\title{
Clinical and biological predictors of response to standardised paediatric colitis therapy (PROTECT): a multicentre inception cohort study
}

\begin{abstract}
JeffreyS Hyams*, Sonia Davis Thomas, Nathan Gotman, Yael Haberman, Rebekah Karns, Melanie Schirmer, Angela Mo, David R Mack, Brendan Boyle, Anne M Griffiths, Neal S LeLeiko, Cary G Saver, David J Keljo, James Markowitz, Susan S Baker, Joel Rosh, Robert N Baldassano, Ashish Patel, Marian Pfefferkorn, Anthony Otley, Melvin Heyman, Joshua Noe, Maria Oliva-Hemker, Paul A Rufo, Jennifer Strople, David Ziring, Stephen L Guthery, Boris Sudel, Keith Benkov, Prateek Wali, Dedrick Moulton, Jonathan Evans, Michael D Kappelman, M Alison Marquis, Francisco A Sylvester, Margaret H Collins, Suresh Venkateswaran, Marla Dubinsky, Vin Tangpricha, Krista L Spada, Bradley Saul, Jessie Wang, Jose Serrano, Kevin Hommel, Urko M Marigorta, Greg Gibson, RamnikJ Xavier, Subra Kugathasan, Thomas Walters, Lee A Denson*
\end{abstract}

\section{Summary}

Lancet 2019; 393: 1708-20 Published Online March 29, 2019 http://dx.doi.org/10.1016/ S0140-6736(18)32592-3 See Comment page 1672

${ }^{*}$ Contributed equally

Division of Digestive Diseases, Hepatology, and Nutrition, Connecticut Children's Medical Center, Hartford, CT, USA (Prof)S Hyams MD, K L Spada BS); Collaborative Studies Coordinating Center (Prof S Davis Thomas DrPH N Gotman MS, M A Marquis MStat, B Saul DrPH, JWang MS), Department of Biostatistics (Prof S Davis Thomas, M D Kappelman MD, Prof F A Sylvester MD), University of North Carolina, Chapel Hill, NC, USA; RTI International, Research Triangle Park, NC, USA (Prof S Davis Thomas); Division of Gastroenterology, Hepatology, and Nutrition, Cincinnati Children's Hospital Medical Center and the University of Cincinnati College of Medicine, Cincinnati, $\mathrm{OH}$, USA (Y Haberman MD, R Karns PhD, Prof M H Collins MD, K Hommel PhD,

Prof LA Denson MD); The Broad Institute of MIT and Harvard, Cambridge, MA, USA

(M Schirmer, Prof RJ Xavier MD); Department of Biostatistics, Harvard T H Chan School of Public Health, Boston, MA, USA (M Schirmer); Georgia Institute of Technology, Atlanta, GA, USA (A Mo BS, U M Marigorta PhD, Prof G Gibson PhD); School of Biological Sciences, Children's Hospital of Eastern Ontario, University of Ottawa, Ottawa, ON, Canada (Prof D R Mack MD); Division of Pediatric

Gastroenterology, Hepatology,

Background Lack of evidence-based outcomes data leads to uncertainty in developing treatment regimens in children who are newly diagnosed with ulcerative colitis. We hypothesised that pretreatment clinical, transcriptomic, and microbial factors predict disease course.

Methods In this inception cohort study, we recruited paediatric patients aged 4-17 years with newly diagnosed ulcerative colitis from 29 centres in the USA and Canada. Patients initially received standardised mesalazine or corticosteroids, with pre-established criteria for escalation to immunomodulators (ie, thiopurines) or anti-tumor necrosis factor-a (TNF $\alpha$ ) therapy. We used RNA sequencing to define rectal gene expression before treatment, and 16S sequencing to characterise rectal and faecal microbiota. The primary outcome was week 52 corticosteroid-free remission with no therapy beyond mesalazine. We assessed factors associated with the primary outcome using logistic regression models of the per-protocol population. This study is registered with ClinicalTrials.gov, number NCT01536535.

Findings Between July 10, 2012, and April 21, 2015, of 467 patients recruited, 428 started medical therapy, of whom $400(93 \%)$ were evaluable at 52 weeks and $386(90 \%)$ completed the study period with no protocol violations $150(38 \%)$ of 400 participants achieved week 52 corticosteroid-free remission, of whom 147 (98\%) were taking mesalazine and three (2\%) were taking no medication. $74(19 \%)$ of 400 were escalated to immunomodulators alone, $123(31 \%)$ anti-TNF $\alpha$ therapy, and 25 (6\%) colectomy. Low baseline clinical severity, high baseline haemoglobin, and week 4 clinical remission were associated with achieving week 52 corticosteroid-free remission $(n=386$, logistic model area under the curve [AUC] $0 \cdot 70,95 \%$ CI $0 \cdot 65-0 \cdot 75$; specificity 77\%, 95\% CI 71-82). Baseline severity and remission by week 4 were validated in an independent cohort of 274 paediatric patients with newly diagnosed ulcerative colitis. After adjusting for clinical predictors, an antimicrobial peptide gene signature (odds ratio [OR] 0.57, 95\% CI 0.39-0.81; $\mathrm{p}=0.002)$ and abundance of Ruminococcaceae (OR 1.43, 1.02-2.00; $\mathrm{p}=0.04)$, and Sutterella (OR 0.81, 0.65-1.00; $\mathrm{p}=0.05$ ) were independently associated with week 52 corticosteroid-free remission.

Interpretation Our findings support the utility of initial clinical activity and treatment response by 4 weeks to predict week 52 corticosteroid-free remission with mesalazine alone in children who are newly diagnosed with ulcerative colitis. The development of personalised clinical and biological signatures holds the promise of informing ulcerative colitis therapeutic decisions.

Funding US National Institutes of Health.

\section{Introduction}

Children diagnosed with ulcerative colitis typically have a moderate-to-severe disease course with high corticosteroid dependency and corticosteroid refractory disease driving frequent escalation to immunomodulators and anti-tumor necrosis factor- $\alpha$ (TNF $\alpha$ ) therapy., ${ }^{1,2} \mathrm{Up}$ to $26 \%$ of children diagnosed with ulcerative colitis have a colectomy within 5 years. ${ }^{1,3}$ We hypothesised that in adults and children the course of ulcerative colitis depends on initial disease phenotype, therapy offered, and intrinsic patient biology.
Initial disease severity, lack of response to early therapy, hypoalbuminaemia, anaemia, low serum concentration of 25-hydroxyvitamin D (25[OH]D), colonic histology, and specific genetic polymorphisms have been associated with worse clinical outcomes; ${ }^{2,46}$ although these data largely come from studies without standardised treatment and many have focused on acutely ill patients being treated in hospital. In a paediatric observational registry with no standardisation of initial therapy, less than $40 \%$ of children who were newly diagnosed with ulcerative colitis 
Research in context

\section{Evidence before this study}

The lack of strong evidence-based outcomes data leads to uncertainty when developing treatment regimens for children who are newly diagnosed with ulcerative colitis, whose disease course might be unpredictable on the basis of initial clinical features alone. Robust controlled clinical trial data are not available in children, and current practice is largely based on extrapolation from adult data. We searched PubMed for studies published in English before Dec 31, 2017, using the MeSH terms "ulcerative colitis", "corticosteroids", "5-aminosalicylates", "treatment", and "outcomes". We identified no systematic prospective studies of initial standardised therapy in children who were treatment naive or large scale microbiome and transcriptomic studies of stool samples and rectal tissue obtained at diagnosis to aid in predicting disease outcome at 1 year. Although studies did identify specific clinical factors associated with treatment outcomes, these factors were limited by small sample sizes, uncontrolled therapies, and lack of replication.

\section{Added value of this study}

Our multicentre study of 428 treatment naive children with ulcerative colitis establishes for the first time, to our knowledge, prospective, controlled outcome data at 1 year after standardised initial therapy with mesalazine or corticosteroid. We developed a clinical model for week 52 corticosteroid-free remission with mesalazine alone that includes baseline clinical and laboratory factors and week 4 response to standardised therapy, and then

were in corticosteroid-free remission without the need for immunomodulators or anti-TNF $\alpha$ therapy 1 year after diagnosis.

The predicting response to standardised paediatric colitis therapy (PROTECT) study was undertaken to provide prospective evidence-based data on the disease course of children who are newly diagnosed with ulcerative colitis and treated with standardised protocols, and identify clinical features and biological pathways that would improve understanding of the pronounced variability in treatment response without the confounding of uncontrolled treatment and retrospective analyses. In PROTECT, we characterised rectal gene expression and the intestinal microbiome before treatment and in association with clinical outcomes to develop predictive models of disease course beyond clinical characteristics.

\section{Methods}

\section{Study design and participants}

In this prospective inception cohort study, we recruited children aged 4-17 years who were undergoing assessment for ulcerative colitis from 29 centres (mostly hospitals) in the USA and Canada. Each child underwent an ileocolonoscopy and oesophagogastroduodenoscopy with biopsy, and a diagnosis of ulcerative colitis was made by use of established criteria. ${ }^{8}$ Patients were eligible if they had disease beyond the rectum, a baseline Pediatric Ulcerative validated the model in an independent cohort. A similar model with high specificity was developed to predict escalation to antitumour necrosis factor- $\alpha$ therapy in our primary cohort. We found that rectal gene expression and gut microbial factors not only improved our ability to predict clinical outcomes beyond initial disease severity and laboratory characteristics, but also provided crucial insight into the biological reasons for widely disparate patient courses. These data highlighted more robust host innate immune responses, and loss of microbial Clostridiales taxa, in worse outcomes after controlling for clinical severity.

\section{Implications of all the available evidence}

Our study has replicated several previous reports of clinical factors linked to outcomes in ulcerative colitis, and for the first time, to our knowledge, developed and validated a clinical model for week 52 corticosteroid-free remission with mesalazine alone in two large paediatric inception cohorts. These results will therefore have an immediate effect on clinical practice by providing specific parameters for initial disease activity, laboratory test results, and histological features, as well as early response to standardised therapy to guide therapeutic decisions. The transcriptomic and microbiome data reveal a possible mechanistic basis for success or failure of response to standardised therapy and the need for treatment escalation. Our data suggest that novel personalised interventions that account for these host-microbe interactions might be beneficial in both guiding more rational use of current therapies and developing novel microbial interventions.

Colitis Activity Index (PUCAI) score of 10 or more, no previous therapy for colitis, and stool culture negative for enteric bacterial pathogens and Clostridium difficile toxin. Exclusion criteria included evidence of Crohn's disease, use of oral corticosteroid within 4 weeks before diagnosis (full inclusion and exclusion criteria are in the appendix).

Each site's institutional review board approved the protocol and safety monitoring plan. Informed consent or assent was obtained for each participant.

\section{Procedures}

Initial therapy was based on disease severity and in cluded mesalazine or oral or intravenous corticosteroids. Standardised treatment guidelines with indications for adding mesalazine, stopping steroids, or escalation to anti-TNF $\alpha$ therapy or immunomodulators (ie, thiopurines) or colectomy are in the appendix.

Clinical and routine laboratory data were collected at diagnosis and weeks 4,12 , and 52, as previously reported. We determined disease activity using PUCAI ${ }^{10}$ and Mayo scores. ${ }^{11}$ Rectal biopsy samples were obtained from the most inflamed part of the rectosigmoid and histology was assessed centrally by a pathologist (MHC) and a histological severity score defined (appendix). ${ }^{12}$ Faecal calprotectin concentration was determined centrally, as previously described. ${ }^{13}$ High density DNA genotyping was done using the Affymetrix UK Biobank Axiom and Nutrition, Nationwide Children's Hospital, Columbus, OH, USA (B Boyle MD); Divisioin of Pediatric Gastroenterology, Hospital For Sick Children, Toronto, ON, Canada (Prof A M Griffiths MD TWalters MD); IBD Centre, Department of Paediatrics, Hasbro Children's Hospital, Providence, RI, USA (Prof N S Leleiko MD); Divisioin of Pediatric Gastroenterology, Nutritiion, and Liver Disease, Emory University, Atlanta, GA, USA (C G Sauer MD,

SVenkateswaran $\mathrm{PhD}$ Prof VTangpricha MD Prof S Kugathasan MD); Division of Gastroenterology, Children's Hospital of Pittsburgh, Pittsburgh, PA, USA (Prof D J Keljo MD); Division of Gastroenterology, Hepatology, and Nutrition, Cohen Children's Medical Center Of New York, New Hyde Park, NY, USA (Prof J Markowitz MD); Division of Pediatric Gastroenterology, Hepatology, and Nutrition, Women \& Children's Hospital of Buffalo WCHOB, Buffalo, NY USA (Prof S S Baker MD); Division of Gastroenterology, Hepatology, and Nutrition, Goryeb Children's Hospital, Atlantic Health, Morristown, NJ, USA (ProfJ Rosh MD); Division of Gastroenterology, Hepatology and Nutrition, The Children's Hospital of Philadelphia, Philadelphia, PA, USA (Prof R N Baldassano MD); Division of Gastroenterology, Hepatology, and Nutrition, UT Southwestern, Dallas, TX, USA (A Patel MD); Division of Gastroenterology, Hepatology, and Nutrition, Riley Children's Hospital Indiana, Indianapolis, IN, USA (M Pfefferkorn MD); Division of Gastroenterology, Hepatology, and Nutrition, IWK Health Centre, Halifax, NS, Canada (Prof A Otley MD); Division of Gastroenterology, Hepatology, and Nutrition, University of California at San Francisco, San Francisco, CA, USA (Prof M Heyman MD); Division of Gastroenterology, Hepatology, and Nutrition, Medical College of Wisconsin, Milwaukee, WI, USA (J Noe MD); Division of Gastroenterology, Hepatology, and Nutrition, Johns Hopkins Children's Center, Baltimore, MD, USA (Prof M Oliva-Hemker MD) Division of Gastroenterology, Hepatology, and Nutrition, 
Children's Hospital Boston, Harvard Medical School Boston, MA, USA (P A Rufo MD); Division of Gastroenterology, Ann \& Robert H Lurie Children's Hospital of Chicago, Chicago, IL, USA (J Strople MD); Division of Gastroenterology, Hepatology, and Nutrition, UCLA Medical Center, Los Angeles, CA, USA (D Ziring MD); Division of Gastroenterology, Hepatology, and Nutrition, Primary

Children's Hospital and the University of Utah, Salt Lake City, UT, USA

(Prof S L Guthery MD); Division of Gastroenterology,

Hepatology, and Nutrition, University of Minnesota

Medical Center, Minneapolis, MN, USA (B Sudel MD); Division of Gastroenterology,

Hepatology, and Nutrition, Mt Sinai Hospital,

New York City, NY, USA (K Benkov MD,

Prof M Dubinsky MD); Division of Gastroenterology,

Hepatology, and Nutrition, State University of New York Upstate Medical University, Syracuse, NY, USA (PWali MD); Division of Gastroenterology, Hepatology, and Nutrition, Monroe Carell Jr Children's Hospital of Vanderbilt, Nashville, TN, USA

(D Moulton MD); Division of Gastroenterology, Hepatology, and Nutrition, Nemours Children's Clinic, Jacksonville,

FL, USA (J Evans MD); National Institutes of Diabetes, Digestive and Kidney Diseases, Bethesda, MD, USA (J Serrano MD); Center for Computational and Integrative Biology,

Gastrointestinal Unit, and Center for the Study of

Inflammatory Bowel Disease, Massachusetts General

Hospital, Boston, MA, USA

(Prof RJ Xavier); Center for

Microbiome Informatics and

Therapeutics, Massachusetts Institute of Technology, Cambridge, MA, USA

(Prof RJ Xavier); and Sheba

Medical Center, affiliated with

the Tel Aviv University, Tel Hashomer, Israel

(Y Haberman)

Correspondence to:

Prof Jeffrey S Hyams, Division of

Digestive Diseases, Hepatology,

and Nutrition, Connecticut

Children's Medical Center,

Hartford, 06106 CT, USA

jhyams@connecticutchildrens.
Array (Thermo-Fisher, Waltham, MA, USA), ${ }^{14}$ and MEMS TrackCap technology (Aardex Group, Seriang, Belgium) was used to monitor mesalamine adherence. RNA sequencing and the global pattern of gene expression of rectal biopsy samples before treatment were determined using RNAseq on the Illumina platform (Illumina, San Diego, CA, USA) ${ }^{15}$ and validated on the Lexogen platform (Lexogen, Vienna, Austria). ${ }^{16}$ Rectal and faecal microbiome was profiled using 16S rRNA amplicon sequencing of the V4 region. ${ }^{17}$ Full details of procedures are in the appendix.

\section{Outcomes}

The primary outcome was week 52 corticosteroid-free remission, defined as clinical remission (PUCAI score of $<10)$ with no corticosteroid use for 4 weeks or longer immediately before week 52 , no medical therapy beyond mesalazine, and no colectomy. The secondary outcome was escalation to anti-TNF $\alpha$ therapy at any time in the 52 weeks. Additional outcomes were escalation to immunomodulators (ie, thiopurine, methotrexate) only, and colectomy. Week 4 remission was defined as a PUCAI score of less than 10 irrespective of corticosteroid status but without immunomodulator or anti-TNF $\alpha$ use, or colectomy. Week 12 corticosteroid-free remission was defined as a PUCAI score of less than 10 at week 12 and corticosteroid-free for the previous 14 days with no previous treatment escalation or colectomy.

We further defined baseline disease severity using a combination of initial PUCAI score and initial therapy as mild (mesalazine, or PUCAI score of $<45$ with oral corticosteroid) or moderate to severe (oral corticosteroid use with PUCAI score of $\geq 45$, or intravenous corticosteroids regardless of PUCAI score) on the basis of previous observations of this cohort, ${ }^{9}$ in which a PUCAI score of less than 45 was identified as the only predictor (odds ratio [OR] 4.38, 95\% CI 1·81-10 · $; \mathrm{p}=0 \cdot 0011$ ), other than remission at week 4 , of corticosteroid-free remission at week 12 in the subgroup starting on oral steroids.

\section{Statistical analysis}

Statistical methods were prespecified in an analysis plan and are fully described in the appendix. We planned a sample size of 430 patients to ensure $90 \%$ power to identify an OR of 2.50 in predictive modelling-a value we expected to be clinically meaningful. Clinical predictors were modelled using the per-protocol population-ie, excluding participants with protocol violations and early study discontinuations with unknown outcomes. For analysis of biological predictors, we restricted sample selection for the primary Illumina platform gene-expression discovery analysis (ie, RNA sequencing) to patients who were treated per-protocol, with mesalazine as the initial therapy for patients with mild disease, and corticosteroids as the initial therapy for patients with moderate-to-severe disease. We aimed to study equal numbers who did or did not achieve week 4 remission because our preliminary data showed that week 4 remission was highly associated with week 52 corticosteroid-free remission. Because of low enrolment of patients with mild disease, and patients with moderateto-severe disease younger than 12 years, we selected all available patients meeting the selection criteria for these groups. Among the moderate-to-severe group aged 12 years and older, we prioritised patients with complete stool sample collection. Statistical tests were done at a two-sided $\alpha$ level of 0.05 with no adjustment for multiple comparisons, unless otherwise specified.

We used an independent prospective inception cohort of children newly diagnosed with ulcerative colitis ${ }^{7}$ to test the clinical model for week 52 corticosteroid-free remission. These patients, diagnosed between 2002 and 2010 at 31 centres in the USA and Canada, prospectively had clinical data obtained at diagnosis, 1 month after diagnosis, and quarterly. Treatment was by provider dictate, not protocol.

We developed prediction models for PROTECT clinical data using multiple imputation multivariable logistic regression with least absolute shrinkage and selection operator $(\mathrm{LASSO})^{18}$ variable selection to account for missing data and obtain a parsimonious model. We defined genes from rectal biopsy samples that were differentially expressed at baseline for patients who were diagnosed with moderate-to-severe disease who achieved and who did not achieve week 52 corticosteroid-free remission with a fold change of 1.5 or higher and a false discovery rate of less than 0.05 . We used ToppGene, ToppCluster, and Cytoscape in gene set enrichment analyses to identify enriched biological pathways within these genes, and we built principal components (PCs) from the gene signatures. We identified differentially abundant microbial organisms associated with week 52 corticosteroid-free remission at a false discovery rate of 0.2 and with escalation to anti-TNF $\alpha$ therapy at a false discovery rate of 0.05 by multivariable analyses accounting for key clinical and demographic covariates. We did a principal coordinates analysis (PcoA) to summarise variation in gene expression between patients, and PC values were extracted for downstream analyses. We tested improvement of clinical prediction models on the addition of each identified gene expression PC or microbial taxon individually, with variables retained at a $\mathrm{p}$ value of less than 0.05 . We then tested the PCs and microbial taxa identified for simultaneous inclusion in the clinical models, in which improvement over the clinical model was assessed via comparison of the area under the receiver operator characteristic (ROC) curve (AUC). We assessed the association between predictors using Spearman correlation.

For the PROTECT main RNA sequence discovery analyses, we included 20 age-matched and sex-matched controls without inflammatory bowel disease (IBD) from a study by Haberman and colleagues ${ }^{19}$ at Cincinnati Children's Hospital Medical Center (Cincinnati, $\mathrm{OH}$, 
USA). For validation of the gene signatures, we used RNA sequence data from an independent cohort, the RISK cohort, ${ }^{20}$ including 43 patients with ulcerative colitis, 93 with Crohn's disease with rectal inflammation, a nd 55 non-IBD controls. The controls we used for the PROTECT and RISK datasets were suspected to have IBD, but with normal radiographic, endoscopic, and histological findings.

We did all statistical testing of predictive models of outcomes using SAS version 9.4. This study is registered with ClinicalTrials.gov (NCT01536535).

\section{Role of the funding source}

The study was performed under a cooperative agreement with the funder, who was involved in the study design, data collection, data analysis, data interpretation, and writing of the report. The corresponding author had full access to all the data in the study and had final responsibility for the decision to submit for publication.

\section{Results}

Between July 10, 2012, and April 21, 2015, 467 patients were enrolled, of whom $36(8 \%)$ were disqualified because of change of diagnosis to Crohn's disease or other protocol violations, and three $(<1 \%)$ patients or their families declined medical therapy. 428 participants started medical therapy, of whom 386 (90\%) were strictly per protocol (figure 1). B aseline c haracteristics f or the w hole c ohort and by disease severity are in table 1 . The mean age was 12.7 years (SD 3.3), $212(50 \%)$ of 428 participants were female, and 69 (16\%) of 420 were non-white (eg, Asian, African American, American Indian, Alaskan Native, Pacific I slander). 178 ( 42\%) p articipants p resented w ith mild disease (mean PUCAI score 31.9 [SD 12.1]; mean total Mayo score $5 \cdot 7$ [SD 1.8]); of whom 136 (76\%) were initially given mesalazine and 42 (24\%) oral corticosteroids. $250(58 \%)$ participants presented with moderate-to-severe disease (mean PUCAI score 62.9 [SD 13.2]; mean total Mayo score $9 \cdot 3$ [SD 1.7]), of whom 102 (41\%) were initially given oral corticosteroids and 148 (59\%) were given intravenous corticosteroids. 286 (67\%) participants received the initial treatment recommended by the standardised treatment algorithm (appendix). By week 52, 387 (90\%) participants received mesalazine at some point, with 293 providing adherence data based on MEMS-Cap use between week 4 and week 52 or escalation to additional therapy. On average, $84 \%$ (SD 21) of prescribed mesalazine doses were taken (82\% [SD 21] by participants with mild disease vs $85 \%$ [SD 22] with moderate-to-severe disease; $\mathrm{p}=0 \cdot 12) .28(6 \%)$ of 428 participants discontinued the study before week 52 without previous colectomy or escalation to immunomodulators or anti-TNF $\alpha$ therapy, leaving 400 (93\%) participants who were evaluable at week 52. An additional 14 patients were excluded from predictive modelling because of protocol violations; hence, the per-protocol population comprised 386 participants. Complete biological data were available for a subset of
177 participants, and 206 had data from RNA sequencing and 343 had microbiome data.

By week 52, 150 (38\%) of 400 participants achieved corticosteroid-free remission (80 [49\%] of 163 with mild disease and 70 [30\%] of 237 with moderate-to-severe disease; appendix). 147 ( $98 \%$ ) of 150 were taking mesalazine and three $(2 \%)$ were taking no medication. 98 (65\%) of 150 participants who achieved remission by week 52 supplied stool samples at that time, of whom 56 (57\%) had faecal calprotectin concentrations below $250 \mathrm{\mu g} / \mathrm{g}$. Of 231 patients who supplied stool samples for analysis at week 52, corticosteroid-free remission with faecal calprotectin concentration below $250 \mu \mathrm{g} / \mathrm{g}$ was achieved by $56(24 \%)$.

Of 400 participants who were evaluable at 52 weeks, $74(19 \%)$ were given immunomodulators as the only additional medical therapy (18 [11\%] of 163 with mild disease and 56 [24\%] of 237 with moderate-to-severe disease) and 123 (31\%) escalated to anti-TNF $\alpha$, including

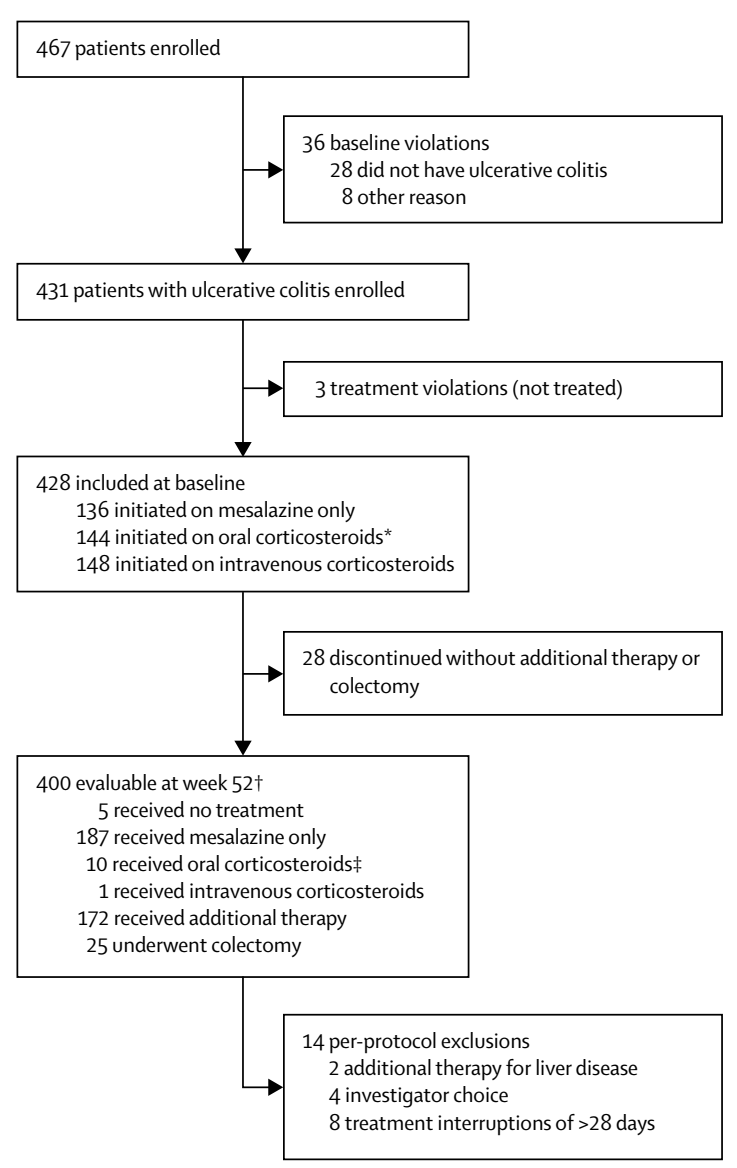

Figure 1: Trial profile

*Three participants were on both oral steroids and mesalazine, and one participant received intravenous steroids for 1 day without being admitted to hospital.

†The evaluable population at week 52 include 17 participants who discontinued after additional therapy (immunomodulator, calcineurin inhibitor, or anti-tumor necrosis factor- $\alpha$ ) or colectomy. ‡Include nine participants on oral corticosteroids and mesalazine. 


\begin{tabular}{|c|c|c|c|c|}
\hline & $\begin{array}{l}\text { Total } \\
(n=428)\end{array}$ & $\begin{array}{l}\text { Mild disease } \\
(n=178)\end{array}$ & $\begin{array}{l}\text { Moderate-to-severe } \\
\text { disease }(n=250)\end{array}$ & p value \\
\hline \multicolumn{5}{|l|}{ Demographic } \\
\hline Age, years & $12 \cdot 7(3 \cdot 3)$ & $12 \cdot 6(3 \cdot 4)$ & $12 \cdot 7(3 \cdot 2)$ & 0.58 \\
\hline \multicolumn{5}{|l|}{ Sex } \\
\hline Female & $212(50 \%)$ & $88(49 \%)$ & $124(50 \%)$ & 0.97 \\
\hline Male & $216(50 \%)$ & $90(51 \%)$ & $126(50 \%)$ &.. \\
\hline Race & $n=420$ & $\mathrm{n}=176$ & $\mathrm{n}=244$ & .. \\
\hline White & $351(84 \%)$ & $150(85 \%)$ & $201(82 \%)$ & 0.44 \\
\hline Non-white & $69(16 \%)$ & $26(15 \%)$ & $43(18 \%)$ & .. \\
\hline Ethnicity & $n=424$ & $\mathrm{n}=175$ & $\mathrm{n}=249$ & .. \\
\hline Hispanic or Latino & $38(9 \%)$ & $21(12 \%)$ & $17(7 \%)$ & 0.066 \\
\hline Not Hispanic or Latino & $386(91 \%)$ & $152(88 \%)$ & $232(93 \%)$ & .. \\
\hline BMI Z score & $-0 \cdot 2(1 \cdot 3)$ & $-0.1(1 \cdot 2)$ & $-0.4(1.4)$ & 0.019 \\
\hline \multicolumn{5}{|l|}{ Initial treatment* } \\
\hline Mesalazine & $136(32 \%)$ & $136(76 \%)$ & 0 & .. \\
\hline Oral corticosteroids & $144(34 \%)$ & $42(24 \%)$ & $102(41 \%)$ & .. \\
\hline Intravenous corticosteroids & $148(35 \%)$ & 0 & $148(59 \%)$ & .. \\
\hline Being treated in hospital at baseline (\%) & $166(39 \%)$ & $9(5 \%)$ & $157(63 \%)$ & $<0.0001$ \\
\hline \multicolumn{5}{|l|}{ Clinical } \\
\hline \multicolumn{5}{|l|}{ Symptoms } \\
\hline Abdominal pain & $354(83 \%)$ & $126(71 \%)$ & $228(91 \%)$ & $<0.0001$ \\
\hline Diarrhoea & $393(92 \%)$ & $144(81 \%)$ & $249(100 \%)$ & $<0.0001$ \\
\hline Rectal bleeding & $398(93 \%)$ & $153(86 \%)$ & $245(98 \%)$ & $<0.0001$ \\
\hline \multicolumn{5}{|l|}{ Disease extent } \\
\hline Proctosigmoiditis & $29(7 \%)$ & $23(13 \%)$ & $6(2 \%)$ & $<0.0001$ \\
\hline Left-sided colitis & $44(10 \%)$ & $34(19 \%)$ & $10(4 \%)$ & .. \\
\hline Extensive disease or pancolitis $\dagger$ & $355(83 \%)$ & $121(68 \%)$ & $234(94 \%)$ & .. \\
\hline $\begin{array}{l}\text { PUCAI total score (range } 0-85 \text { in } \\
\text { increments of } 5 \text { ) } \dagger\end{array}$ & $50 \cdot 0(19 \cdot 9)$ & $31 \cdot 9(12 \cdot 1)$ & $62 \cdot 9(13 \cdot 2)$ & $<0.0001$ \\
\hline$<45$ & $156(36 \%)$ & $148(83 \%)$ & $8(3 \%)$ & $<0.0001$ \\
\hline Mayo total score (range 0-12) & $7 \cdot 8(2 \cdot 5)$ & $5 \cdot 7(1 \cdot 8)$ & $9 \cdot 3(1 \cdot 7)$ & $<0.0001$ \\
\hline$\geq 10$ & $120(28 \%)$ & $3(2 \%)$ & $117(47 \%)$ & $<0.0001$ \\
\hline$\geq 11$ & $71(17 \%)$ & $0(0 \%)$ & $71(28 \%)$ & $<0.0001$ \\
\hline Mayo endoscopy sub-score (range 0-3) & $2 \cdot 2(0 \cdot 7)$ & $1.9(0.6)$ & $2 \cdot 4(0 \cdot 6)$ & $<0.0001$ \\
\hline Partial Mayo score (range 0-9) & $5 \cdot 6(2 \cdot 1)$ & $3.9(1.5)$ & $6 \cdot 8(1.4)$ & $<0.0001$ \\
\hline Haemoglobin, g/dL & $\mathrm{n}=402$ & $\mathrm{n}=163$ & $\mathrm{n}=239$ & .. \\
\hline Mean & $11 \cdot 4(2 \cdot 2)$ & $12 \cdot 2(1 \cdot 9)$ & $10 \cdot 9(2 \cdot 2)$ & $<0.0001$ \\
\hline$<10$ & $98(24 \%)$ & $15(9 \%)$ & $83(35 \%)$ & .. \\
\hline Erythrocyte sedimentation rate, $\mathrm{mm} / \mathrm{h}$ & $\mathrm{n}=385$ & $\mathrm{n}=155$ & $\mathrm{n}=230$ & .. \\
\hline Median & $25(12-42)$ & $16(9-28)$ & $32(17-49)$ & $<0.0001$ \\
\hline $\begin{array}{l}\text { C-reactive protein or high sensitivity } \\
\text { C-reactive protein }\end{array}$ & $\mathrm{n}=315$ & $\mathrm{n}=121$ & $\mathrm{n}=194$ & .. \\
\hline$>U L N$ & $144(46 \%)$ & $36(30 \%)$ & $108(56 \%)$ & $<0.0001$ \\
\hline$>2 \times U L N$ & $97(31 \%)$ & $19(16 \%)$ & $78(40 \%)$ & $<0.0001$ \\
\hline Albumin, $\mathrm{g} / \mathrm{dL}$ & $n=422$ & $\mathrm{n}=174$ & $\mathrm{n}=248$ & .. \\
\hline Mean & $3 \cdot 7(0 \cdot 7)$ & $3 \cdot 9(0 \cdot 7)$ & $3 \cdot 5(0 \cdot 7)$ & $<0.0001$ \\
\hline$<3.5$ & $138(33 \%)$ & $37(21 \%)$ & $101(41 \%)$ &.$\cdot$ \\
\hline Serum 25-hydroxyvitamin $\mathrm{D}, \mathrm{ng} / \mathrm{mL}$ & $n=393$ & $n=163$ & $\mathrm{n}=230$ & .. \\
\hline Median & $\begin{array}{l}28 \cdot 5 \\
(23 \cdot 9-34 \cdot 8)\end{array}$ & $\begin{array}{l}28 \cdot 6 \\
(24 \cdot 3-34 \cdot 1)\end{array}$ & $\begin{array}{l}28 \cdot 3 \\
(23 \cdot 6-35 \cdot 0)\end{array}$ & 0.96 \\
\hline$<20$ & $42(11 \%)$ & $17(10 \%)$ & $25(11 \%)$ & $0 \cdot 70$ \\
\hline $20-30$ & $183(46 \%)$ & $80(49 \%)$ & $103(45 \%)$ & .. \\
\hline$\geq 30$ & $168(43 \%)$ & $66(40 \%)$ & $102(44 \%)$ & .. \\
\hline
\end{tabular}

one patient who escalated to calcineurin inhibitor and one who received an emergent colectomy before antiTNF $\alpha$ therapy (28 (17\%) with mild disease and 95 [40\%] with moderate-to-severe disease). 25 (6\%) of 400 participants had a colectomy (two (1\%) with mild disease and $23(10 \%)$ with moderate-to-severe disease). The timecourse of treatments is shown in the appendix.

Figure 2 shows time until corticosteroid-free remission and escalation to anti-TNF $\alpha$ by initial disease presentation. Participants who presented with mild disease continued to achieve corticosteroid-free remission from week 4 until week 52, whereas those who presented with moderate-to-severe disease and who achieved corticosteroid-free remission had largely done so by week 16 . Of the 95 participants with moderate-to-severe disease who escalated to anti-TNF $\alpha$ therapy, 41 (43\%) had done so by week 4 (figure $2 \mathrm{~B}$ ). After week 4 , participants with mild disease and with moderate-to-severe disease escalated to anti-TNF $\alpha$ therapy at similar incidences. At week 52, $50(48 \%)$ of 105 participants who escalated to anti-TNF $\alpha$ therapy and had 1 year of follow-up were in clinical remission (table 2). Time to corticosteroid-free remission, escalation to immunomodulators, anti-TNF $\alpha$ therapy, and colectomy as a function of remission status at week 4 are shown in the appendix. Of 211 participants who did not achieve remission by week 4, 21 (10\%) had by week 16 ; however, few ultimately reached corticosteroidfree remission by week 52 (50 [24\%] of 211). Conversely, $38(18 \%)$ of 211 who achieved week 4 remission escalated to anti-TNF $\alpha$ primarily between weeks 16 and 52 .

Baseline characteristics, remission by week 4, corticosteroid-free remission by week 12 , and week 52 PUCAI scores by outcome at week 52 are shown in table 2 (additional baseline characteristics, and for the moderateto-severe subgroup, are in the appendix). Incidence of outcomes did not vary with age, sex, or race. As expected, patients who achieved corticosteroid-free remission by week 52 were more likely to have mild disease as determined by clinical and endoscopic analysis, higher haemoglobin and albumin concentrations, lower acute phase reactants (ie, $\mathrm{C}$ reactive protein and erythrocyte sedimenation rate), and higher incidences of remission by week 4 and week 12 than those who did not achieve corticosteroid-free remission by week 52 . However, substantial variability was seen, with 41 (31\%) of 133 participants with a baseline PUCAI score of 65 of higher achieving week 52 corticosteroid-free remission, and $13(14 \%)$ of 90 participants with a PUCAI score below 35 escalated to anti-TNF $\alpha$ therapy by week 52 (table 2). We observed that the bioavailable fraction of $25(\mathrm{OH}) \mathrm{D}$ was higher in those who achieved week 52 corticosteroid-free remission than those who did not achieve 52 week corticosteroid-free remission (median $1.5 \mathrm{ng} / \mathrm{mL}$ vs $1.2-1.3 \mathrm{ng} / \mathrm{mL} ; \mathrm{p}=0.0091$; table 2 ). In participants with moderate-to-severe disease, serum 25(OH)D concentration and bioavailable fraction were lower among those who escalated to anti-TNF $\alpha$ therapy 
(median $26.6 \mathrm{ng} / \mathrm{mL}$ serum and $1.1 \mathrm{ng} / \mathrm{mL}$ bioavailable fraction) than those not escalated to anti-TNF $\alpha$ therapy (median 28.7-30.6 ng/mL for serum, $\mathrm{p}=0.026$; median for bioavailable $1 \cdot 1-1 \cdot 3 \mathrm{ng} / \mathrm{mL}, \mathrm{p}=0 \cdot 055$; appendix). Patients who escalated to anti-TNF $\alpha$ were less likely to have increased concentrations of eosinophils on rectal histopathological assessment than those who did not escalate to anti-TNF $\alpha$ therapy (45 [42\%] of 107 vs $60-68 \%$, $\mathrm{p}=0.0016$; table 2); this observation was particularly apparent in those with moderate-to-severe disease (31 [37\%] of 83 vs 54-74\%, p=0 - 0004; appendix). Mesalazine adherence was not associated with achieving week 52 corticosteroid-free remission or escalation to anti-TNF $\alpha$ therapy (appendix). Week 4 remission and week 12 corticosteroid-free remission were both strongly associated with outcomes at week 52, and had a modest correlation (Spearman correlation 0.40, 95\% CI 0.31-0.48; p<0 . 0001) in the week 52 evaluable population $(n=400)$.

We identified a panel of 33 genes that were differentially expressed in the rectum for patients with moderate-to-severe disease who did $(n=51)$ and did not $(\mathrm{n}=101)$ achieve week 52 corticosteroid-free remission (figure 3; appendix). We identified 18 gene sets that were increased, which were associated with cellular transport and channels, and 15 gene sets that were decreased and associated with antimicrobial responses (more information is in the appendix). From the 206 participants who had RNA sequencing data, unsupervised hierarchal clustering of the gene panel was defined in 199 patients with ulcerative colitis (block 1 [n=3], blocks ii-a [n=79] and ii-b [ $\mathrm{n}=117]$, full gene panel is in the appendix; figure $3 \mathrm{~A}$ ). Participants who achieved week 52 corticosteroidfree remission were more likely to be in block ii-b (52 [69\%] of 75 participants who achieved corticosteroidfree remission in block ii; $\mathrm{p}=0 \cdot 03$ ), whereas those who escalated to anti-TNF $\alpha$ were more likely to be in block ii-a (42 [66\%] of 64 who escalated to anti-TNF $\alpha$ in block ii; $\left.p=1 \times 10^{-6}\right)$. Gene set enrichment analysis of the gene panel identified epithelial transporters and channels, and antimicrobial peptides including $\alpha$-defensins (the transport and antimicrobial gene signature; figure $3 \mathrm{~A}$ ). The $\alpha$-defensins reactome antimicrobial peptide pathway showed a stronger negative association with week 52 corticosteroid-free remission outcome (table 3), whereas the overall 33 gene set signature was negatively associated with need for escalation to anti-TNF $\alpha$ therapy (table 4). A specific antimicrobial peptide pathway was induced in ulcerative colitis compared with 20 age-matched and sex-matched controls (appendix), but in relatively lower concentrations than in many of the patients who achieved week 52 corticosteroid-free remission (appendix). Consistent with this number, a greater number of $\alpha$-defensin 5 (DEFA5) positive cells was detected in rectal biopsy samples from patients with ulcerative colitis who did not achieve week 52 corticosteroid-free remission than in samples from those who did and controls (appendix). By comparison, the

\begin{tabular}{|c|c|c|c|c|}
\hline & $\begin{array}{l}\text { Total } \\
(n=428)\end{array}$ & $\begin{array}{l}\text { Mild disease } \\
(n=178)\end{array}$ & $\begin{array}{l}\text { Moderate-to-severe } \\
\text { disease }(n=250)\end{array}$ & $p$ value \\
\hline \multicolumn{5}{|l|}{ (Continued from previous page) } \\
\hline $\begin{array}{l}\text { Bioavailable 25-hydroxyvitamin D, } \\
\mathrm{ng} / \mathrm{mL}\end{array}$ & $n=393$ & $n=163$ & $n=230$ &. \\
\hline Median & $\begin{array}{c}1.33 \\
(0.83-1.93)\end{array}$ & $\begin{array}{c}1.55 \\
(1 \cdot 08-2 \cdot 16)\end{array}$ & $\begin{array}{c}1 \cdot 17 \\
(0 \cdot 75-1 \cdot 74)\end{array}$ & $<0.0001$ \\
\hline$\geq 1 \cdot 6$ & $147(38 \%)$ & $79(48 \%)$ & $68(30 \%)$ & 0.00021 \\
\hline $\begin{array}{l}\text { Rectal biopsy eosinophilic } \\
\text { inflammation, count }>32 \text { per hpf }\end{array}$ & $\begin{array}{l}210 / 367 \\
(57 \%)\end{array}$ & $\begin{array}{l}92 / 151 \\
(61 \%)\end{array}$ & $\begin{array}{l}118 / 216 \\
(55 \%)\end{array}$ & 0.23 \\
\hline
\end{tabular}

Data are mean (SD), $\mathrm{n}(\%), \mathrm{n} / \mathrm{N}(\%)$, or median (IQR). $\mathrm{p}$ values comparing groups are from a $\chi^{2}$ test for categorical variables, a Mantel-Haenszel $\chi^{2}$ test for ordinal variables, and ANOVA for continuous variables. Additional characteristics are in the appendix. BMI=body-mass index. PUCAl=Pediatric Ulcerative Colitis Activity Index. ULN=upper limit of normal. $\mathrm{hpf}=$ high power field. *Some participants received a different initial treatment from the recommended plan at the discretion of the treating physician. Includes patients with fulminant disease who were limited to colonoscopy

Table 1: Demographic and clinical characteristics of study population by disease presentation

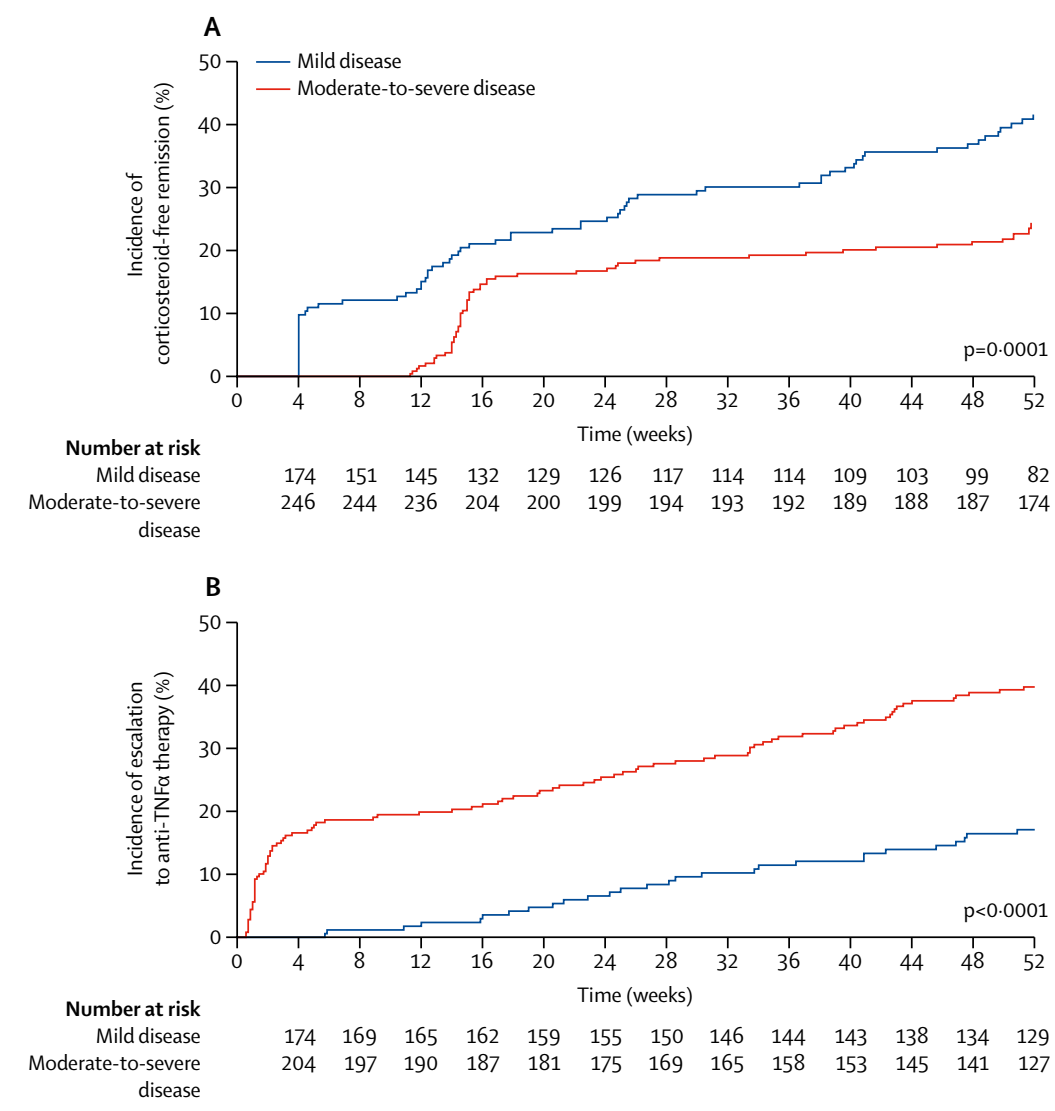

Figure 2: Time until corticosteroid-free remission (A) or escalation to anti-TNF $\alpha$ therapy (B) by initial disease presentation

Data are from a Kaplan-Meier analysis for all 428 participants who started therapy, of whom 178 presented with mild disease and 250 with moderate-to-severe disease. Time until event was censored at last contact for

28 patients who discontinued before the week 52 study visit without treatment escalation. Differences between groups were tested by log-rank test.

epithelial transport gene signature was suppressed in patients with ulcerative colitis compared with controls, and suppression was greatest in patients who escalated 
Week 52

corticosteroid-free remission $(n=150)$
Neither corticosteroid- Escalation to

free remission nor additional therapy $(n=53)$

\section{Demographic}

Age, years

Sex

Female

Male

Race

White

Non-white

Clinical

Extent of disease

Proctosigmoiditis

Left-sided colitis

Extensive disease or pancolitisł

PUCAl total score (range $0-85$ by increments of 5)

$\begin{array}{cc}12 \cdot 3(3 \cdot 4) & 12 \cdot 4(3 \cdot 8) \\ 69(46 \%) & 30(57 \%) \\ 81(54 \%) & 23(43 \%) \\ \mathrm{n}=148 & \mathrm{n}=53 \\ 126(85 \%) & 44(83 \%) \\ 22(15 \%) & 9(17 \%)\end{array}$

immunomod

only* $(n=74)$

therapy $\dagger$
$(n=123)$

$\begin{array}{rc}15(10 \%) & 5(9 \%) \\ 18(12 \%) & 8(15 \%) \\ 117(78 \%) & 40(75 \%)\end{array}$

$6(8 \%)$

$65(88 \%)$

$39 \cdot 2(16 \cdot 3)$

$51.6(15.4)$

$12 \cdot 9(2 \cdot 9)$
$36(49 \%)$
$38(51 \%)$
$n=70$
$59(84 \%)$
$11(16 \%)$

Proportion of week 52 outcomes by PUCAI total score category

$10-30$ (mild) $\mathrm{n}=90$
$35-60$ (moderate) $\mathrm{n}=177$
$\geq 65$ (severe) $\mathrm{n}=133$
Mayo total score (range $0-12$ )
$\geq 10$
$\geq 11$
Mayo endoscopy score (range $0-3$ )
Haemoglobin, g/dL
Mean
$<10$

$34(64 \%)$

$43 / 90(48 \%)$

$66 / 177(37 \%)$

$41 / 133(31 \%)$

$7 \cdot 2(2 \cdot 5)$

$30(20 \%)$

$13(9 \%)$

$2 \cdot 1(0 \cdot 7)$

$\mathrm{n}=139$

$11 \cdot 9(2 \cdot 0)$

$22(16 \%)$

Erythrocyte sedimentation rate, $\mathrm{mm} / \mathrm{h} \quad \mathrm{n}=135$

Median

$19(11-34)$

C-reactive protein or high sensitivity

C-reactive protein

$>$ ULN

Mean

$<3.5$

Serum 25-hydroxyvitamin D, ng/mL

Median

$<20$

$20-<30$

$\geq 30$

Bioavailable 25-hydroxyvitamin D,

$\mathrm{ng} / \mathrm{mL}$

Median

$\geq 1 \cdot 6$

Rectal biopsy eosinophilic

inflammation, count $>32 / \mathrm{hpf}$

Week 4 remission $§$

Week 4 faecal calprotectin, $\mu \mathrm{g} / \mathrm{g}$

Median

Week 12 corticosteroid-free remission

$$
\mathrm{n}=113
$$

$42(37 \%)$

$\mathrm{n}=147$

$3.8(0 \cdot 7)$

$40(27 \%)$

$\mathrm{n}=136$

$29 \cdot 5(25 \cdot 0-36 \cdot 9)$

$12(9 \%)$

$61(45 \%)$

$63(46 \%)$

$\mathrm{n}=136$

$$
\begin{aligned}
& 1 \cdot 5(1 \cdot 1-2 \cdot 1) \\
& 65(48 \%) \\
& 75 / 126(60 \%)
\end{aligned}
$$

$100 / 150(67 \%)$

$\mathrm{n}=108$

$604 \cdot 5(144 \cdot 8-1519 \cdot 3)$

$79 / 150(53 \%)$

$23 / 90(26 \%)$
$25 / 177(14 \%)$
$5 / 133(4 \%)$
$6 \cdot 5(2 \cdot 0)$
$6(11 \%)$
$1(2 \%)$
$1 \cdot 9(0 \cdot 6)$
$\mathrm{n}=49$
$12 \cdot 0(1 \cdot 9)$
$7(14 \%)$

$\mathrm{n}=44$

17 (9-35)

$n=37$

$$
12(32 \%)
$$

$\mathrm{n}=53$

$3.8(0.8)$

$15(28 \%)$

$\mathrm{n}=50$

$28.8(24 \cdot 2-34 \cdot 0)$

$5(10 \%)$

$23(46 \%)$

$22(44 \%)$

$n=51$

\section{$1 \cdot 3(0 \cdot 8-2 \cdot 1)$ \\ 20 (39\%)}

$31 / 46(67 \%)$

$27 / 53(51 \%)$

$\mathrm{n}=39$

677.6 (233.2-1597.7)

$23 / 53(43 \%)$
$16(22 \%)$

$12 \cdot 9(3 \cdot 0)$
$62(50 \%)$
$61(50 \%)$
$\mathrm{n}=121$
$101(83 \%)$
$20(17 \%)$

$0 \cdot 30$

0.60

..

0.98

..

0.0041

$4(3 \%)$

$6(5 \%)$

$113(92 \%)$

$60 \cdot 2(18 \cdot 1)$

..

..

$<0.0001$

$23(19 \%)$

$<0.0001$

$11 / 90(12 \%)$

45/177 (25\%)

$18 / 133(14 \%)$

$7.9(2.0)$

$15(20 \%)$

$8(11 \%)$

$2 \cdot 2(0.6)$

$\mathrm{n}=68$

$10.9(2 \cdot 3)$

$22(32 \%)$

$\mathrm{n}=64$

28 (12-47)

$n=51$

$24(47 \%)$

$\mathrm{n}=71$

$3.6(0.6)$

$22(31 \%)$

$\mathrm{n}=68$

$28 \cdot 7(24 \cdot 4-35 \cdot 1)$

$3(4 \%)$

$34(50 \%)$

$31(46 \%)$

$\mathrm{n}=66$

$1 \cdot 2(0 \cdot 8-1 \cdot 8)$
$21(32 \%)$
$43 / 63(68 \%)$
$33 / 74(45 \%)$
$\mathrm{n}=48$
$1014 \cdot 3(372 \cdot 0-2314 \cdot 2)$
$15 / 74(20 \%)$

$13 / 90(14 \%)$

41/177 (23\%)

69/133 (52\%)

$9 \cdot 1(2 \cdot 3)$

$64(52 \%)$

$47(38 \%)$

$2.5(0.6)$

$\mathrm{n}=120$

$10 \cdot 8(2 \cdot 3)$

45 (38\%)

$\mathrm{n}=115$

30 (17-46)

$\mathrm{n}=100$

$59(59 \%)$

$\mathrm{n}=123$

$3.5(0 \cdot 7)$

$55(45 \%)$

$\mathrm{n}=113$

$27 \cdot 1(22 \cdot 2-31 \cdot 7)$

$19(17 \%)$

$54(48 \%)$

$40(35 \%)$

$\mathrm{n}=112$

\section{$1.2(0 \cdot 8-1 \cdot 9)$}

$36(32 \%)$

45/107 (42\%)

$<0.0001$

..

$<0.0001$

$<0.0001$

$<0.0001$

$<0.0001$

$<0.0001$

0.0001

..

0.0005

$38 / 123(31 \%) \quad<0.0001$

$\mathrm{n}=78$

$1576.8(564.6-2975 \cdot 5) \quad 0.0008$ $19 / 123(15 \%) \quad<0.0001$

(Table 2 continues on next page) 


\begin{tabular}{|c|c|c|c|c|c|}
\hline & $\begin{array}{l}\text { Week } 52 \\
\text { corticosteroid-free } \\
\text { remission }(n=150)\end{array}$ & $\begin{array}{l}\text { Neither corticosteroid- } \\
\text { free remission nor } \\
\text { additional therapy } \\
(n=53)\end{array}$ & $\begin{array}{l}\text { Escalation to } \\
\text { immunomodulator } \\
\text { only }^{*}(n=74)\end{array}$ & $\begin{array}{l}\text { Escalation to anti-TNF } \alpha \\
\text { therapy }+(n=123)\end{array}$ & $p$ value \\
\hline \multicolumn{6}{|l|}{ (Continued form previous page) } \\
\hline Week 52 PUCAI total score category & $\mathrm{n}=149$ & $n=51$ & $n=69$ & $\mathrm{n}=105$ &.. \\
\hline$<10$ (inactive) & $149(100 \%)$ & $6(12 \%)$ & $40(58 \%)$ & $50(48 \%)$ & $<0.0001$ \\
\hline $10-30$ (mild) & 0 & $39(76 \%)$ & $24(35 \%)$ & $32(30 \%)$ &.$\cdot$ \\
\hline 35-60 (moderate) & 0 & $5(10 \%)$ & $3(4 \%)$ & $17(16 \%)$ &.$\cdot$ \\
\hline$\geq 65$ (severe) & 0 & $1(2 \%)$ & $2(3 \%)$ & $6(6 \%)$ & .. \\
\hline
\end{tabular}

Data are mean (SD), $\mathrm{n}(\%), \mathrm{n} / \mathrm{N}(\%)$, or median (IQR). Evaluable population excludes participants who discontinued the study without additional therapy or colectomy. $\mathrm{p}$ values comparing groups are from a $\chi^{2}$ testfor categorical variables, a Mantel-Haenszel $\chi^{2}$ test for ordinal variables, and ANOVA or Kruskal-Wallis test for continuous variables. Additional characteristics are in the appendix. PUCAl=Pediatric Ulcerative Colitis Activity Index. TNF=tumor necrosis factor. ULN=upper limit of normal. hpf=high power field. ${ }^{*} 106$ patients started on immunomodulators, 32 later escalated to anti-TNFa. Includes one patient starting a calcineurin inhibitor and one who received colectomy without escalation to

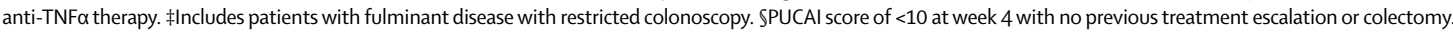

Table 2: Baseline characteristics, week 4 remission, week 12 corticosteroid-free remission, and week 52 PUCAI score of the evaluable population by week 52 outcome

to anti-TNF $\alpha$ therapy (appendix). A specific induction of the antimicrobial peptide gene signature and suppression of the epithelial transport gene signature were confirmed in patients with ulcerative colitis compared with non-IBD controls and controls with Crohn's disease involving the rectum in the independent RISK cohort (appendix). ${ }^{20}$

Microbes from the Clostridiales order have been implicated in maintenance of the function of the gut barrier and immune tolerance. ${ }^{21}$ Multivariable analyses identified taxa associated with week 52 corticosteroid-free remission (six at a false discovery rate of $<0 \cdot 2$, with two at a false discovery rate of $<0.05$ ), and escalation to antiTNF $\alpha$ therapy ( 56 at a false discovery rate of $<0 \cdot 2$, with 26 at a false discovery rate of $<0 \cdot 05$; appendix). Almost all of these taxa were increased in patients who achieved corticosteroid-free remission at week 52 , and decreased in those who escalated to anti-TNF $\alpha$ therapy. These taxa included two operational taxonomic units from the Clostridiales order that were increased in patients who achieved week 52 corticosteroid-free remission, and 20 Clostridiales operational taxonomic units that were decreased in patients who escalated to anti-TNF $\alpha$ therapy (false discovery rate of $<0 \cdot 05$; appendix).

Mild clinical severity (PUCAI score of $<45$, regardless of initial treatment), week 4 remission, and baseline haemoglobin concentration of $10 \mathrm{~g} / \mathrm{dL}$ or higher in those who did not achieve remission by week 4 were associated with achieving week 52 corticosteroid-free remission (table 3). The multivariable model of the per-protocol population $(\mathrm{n}=386)$ showed good discriminatory power and specificity, with an AUC of $0 \cdot 70$ (95\% CI 0.65-0 - 75), a cross-validated AUC of $0.63(0.57-0.69)$, and specificity of $77 \%$ (95\% CI 71-82); table 3, appendix). A post-hoc model with week 12 corticosteroid-free remission instead of week 4 remission had a similar discriminatory power, with an AUC of 0.69 (95\% CI 0.64-0.75) and specificity of $77 \%$ (95\% CI 77-82; appendix).
Components of the clinical model were validated in an independent inception cohort of 307 paediatric ulcerative colitis patients enrolled in the prospective Pediatric Inflammatory Bowel Disease Collaborative Research Group Registry (appendix). . Baseline clinical and demographic characteristics were quite similar between the IBD registry study and the PROTECT cohort. Disease activity of this independent cohort was classified using physician global assessment because enrolment occurred before the PUCAI was published. ${ }^{10}$ Medical therapy was not standardised, and 86 (28\%) of 307 patients achieved week 52 corticosteroid-free remission. Multivariable analysis in the 274 (89\%) of 307 participants with complete data confirmed that mild clinical disease activity at baseline (OR 1.7, 95\% CI $1 \cdot 0-3 \cdot 0 ; \mathrm{p}=0 \cdot 056)$ and week 4 inactive disease $(2 \cdot 8$, $1.7-4.8 ; \quad p=0.0001)$ were associated with week 52 corticosteroid-free remission, with similar discriminatory power (AUC $0 \cdot 65$, 95\% CI 0.59-0.71). Baseline haemoglobin concentration did not achieve significance, but the direction of effect was consistent with the PROTECT clinical model (continuous haemoglobin OR $1 \cdot 08 / 1$-unit increase; $\mathrm{p}=0 \cdot 18$; haemoglobin $\geq 10 \mathrm{~g} / \mathrm{dL}$ OR $1 \cdot 55 ; \mathrm{p}=0 \cdot 19)$.

The association between the probability of achieving week 52 corticosteroid-free remission based upon the PROTECT clinical model and the antimicrobial peptide gene signature is shown the appendix. Patients with a lower antimicrobial peptide gene signature single geneexpression PC (PC1) were more likely to achieve week 52 corticosteroid-free remission than those with a higher concentrations of PC1. The addition of biological data on antimicrobial peptide gene signature and the relative abundance of Ruminococcaceae and Sutterella organisms to the clinical model improved the discriminantory power of the model, with an AUC of 0.75 (95\% CI 0.68-0.83; $\mathrm{p}=0.014)$, a cross-validated AUC of $0.72 \quad(95 \%$ CI $0 \cdot 64-0 \cdot 80)$, and specificity of $78 \%(95 \%$ CI $70-85)$ in 
A

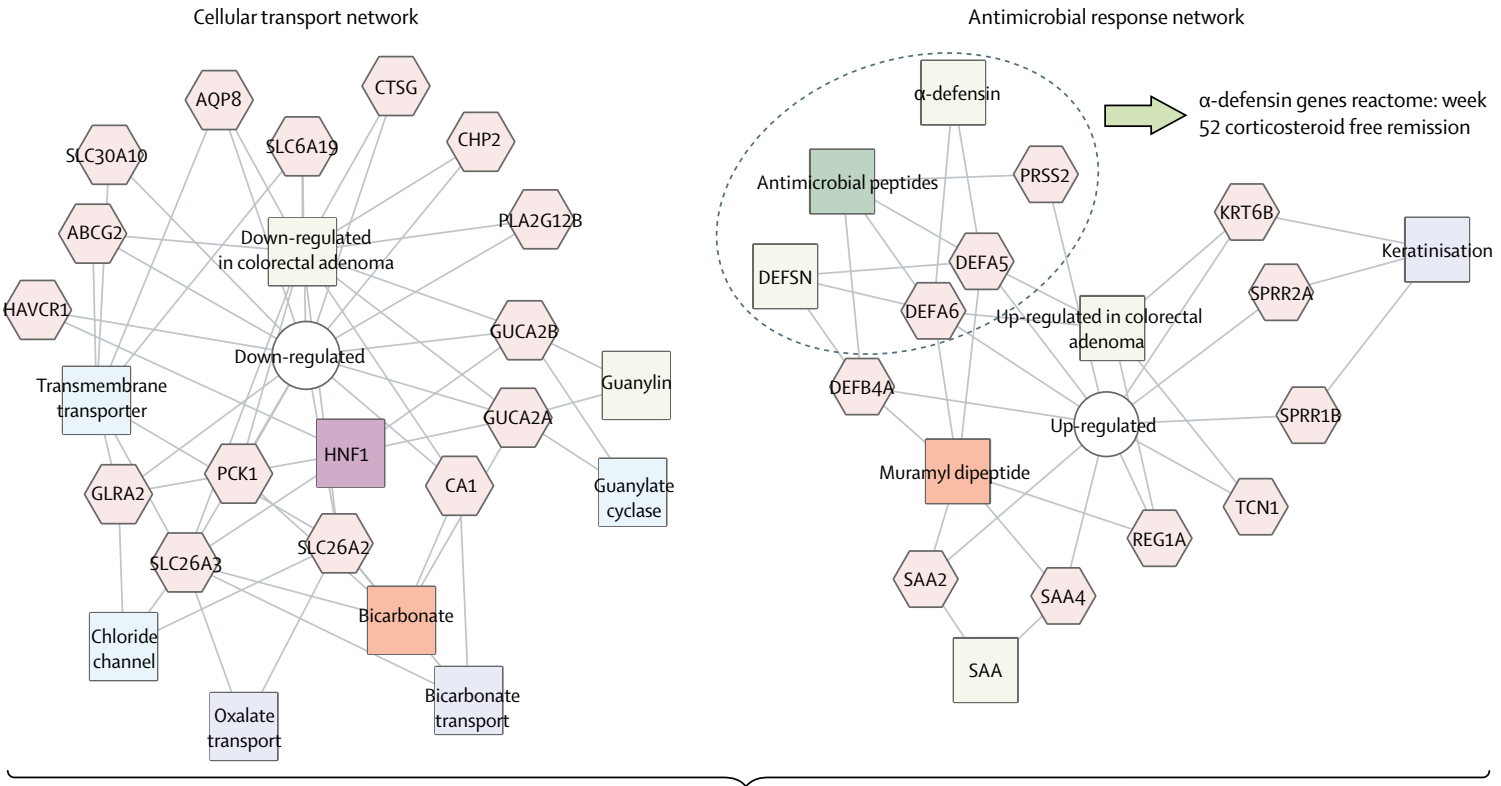

Escalation to anti-TNF $\alpha$ therapy or colectomy
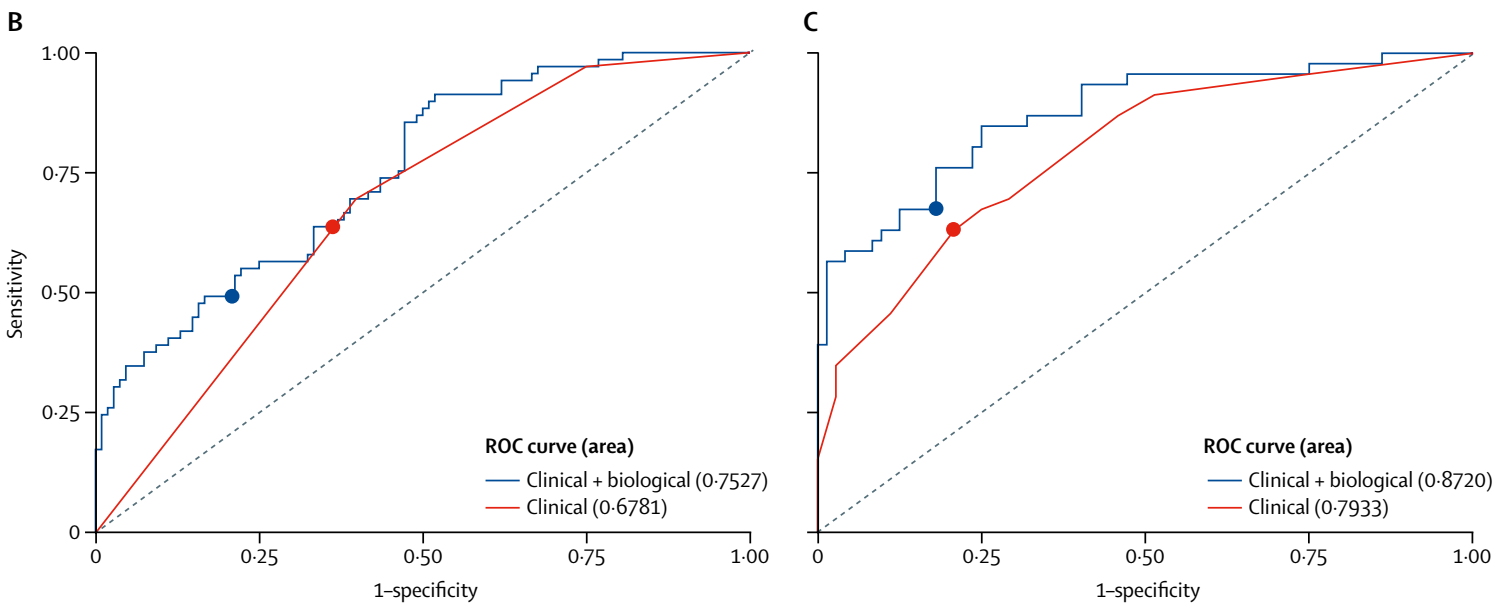

Figure 3: Gene set enrichment analysis (A) and receiver operator characteristic curves for week 52 corticosteroid-free remission (B) and escalation to anti-TNF $\alpha$ therapy or $(C)$ colectomy as biological predictors of disease outcome

(A) 33 genes were differentially expressed in the rectum in participants with moderate-to-severe disease who achieved week 52 corticosteroid-free remission compared with those who did not. 18 increased genes (15 shown) were associated with cellular transport and channel, and 15 decreased gene sets (11 shown) were associated with antimicrobial responses (more information is in the appendix). Genes are shown as hexagons, gene ontology as blue squares, drug-associations as orange squares, and pathways, gene families, interactions, and co-expression are in different shades of green. The full list of results and $p$ values are in the appendix. (B and C) ROC curves of outcomes from logistic regression models of patients with clinical data (red) and clinical and biological data (blue) from single imputation with the median AUC value out of the 100 multiple imputations. Panel B shows data for the whole cohort and panel C for patients with moderate-to-severe disease. The cutpoint used to define sensitivity and specificity for each model is indicated by the filled circle. Panel B corresponds to data in table 3 and panel $C$ to data in table 4 . AUC=area under the curve. $\mathrm{ROC}=$ receiver operator characteristic.

the subset with transcriptomic and microbial data $(\mathrm{n}=177$; figure 3B). Patients with lower levels of antimicrobial peptide gene expression (OR 0.57, 95\% CI $0 \cdot 39-0.81 ; \mathrm{p}=0.002)$ and Sutterella organisms $(0 \cdot 81$, $0 \cdot 65-1 \cdot 00 ; \mathrm{p}=0 \cdot 05)$ and higher relative abundance of Ruminococcaceae $(1 \cdot 43,1 \cdot 02-2 \cdot 00 ; \mathrm{p}=0 \cdot 04)$, were more likely to achieve week 52 corticosteroid-free remission than other patient groups.
Because 95 (40\%) of 237 evaluable patients with moderate-to-severe disease escalated to anti-TNFa therapy by week 52 (appendix), we sought to define factors associated with this outcome. In the per-protocol population with moderate-to-severe disease $(n=232)$, a total Mayo score of 11 or more and lack of remission by week 4 were associated with escalation to anti-TNFa therapy (table 4). Notably, baseline haemoglobin 


\begin{tabular}{|c|c|c|c|c|c|c|}
\hline & \multicolumn{2}{|c|}{$\begin{array}{l}\text { All patients in clinical model } \\
(n=386 ; 147[38 \%] \text { events })\end{array}$} & \multicolumn{4}{|c|}{ Patients with biological data $\nmid(n=177 ; 69[39 \%]$ events) } \\
\hline & \multirow[t]{2}{*}{ Estimate } & \multirow[t]{2}{*}{ p value } & \multicolumn{2}{|l|}{ Clinical model } & \multicolumn{2}{|c|}{ Clinical and biological model } \\
\hline & & & Estimate & p value & Estimate & p value \\
\hline \multicolumn{7}{|l|}{ Baseline predictors } \\
\hline PUCAI score $<45$ & $1.84(1 \cdot 18-2 \cdot 89)$ & 0.0077 & NA & & NA &.. \\
\hline Haemoglobin $\geq 10 \mathrm{~g} / \mathrm{dL}$ (without week 4 remission) & $4 \cdot 24(1 \cdot 46-12 \cdot 31)$ & 0.0080 & $6 \cdot 79(1.45-31 \cdot 76)$ & 0.015 & $5 \cdot 72(1.16-28 \cdot 23)$ & 0.032 \\
\hline Week 4 remission & $9 \cdot 50(3 \cdot 39-26 \cdot 63)$ & $<0.0001$ & $15 \cdot 11(3 \cdot 39-67 \cdot 44)$ & 0.00037 & $14.92(3 \cdot 18-69 \cdot 98)$ & 0.00061 \\
\hline Antimicrobial peptide gene signature & NA & .. & NA &.. & $0.57(0.39-0.81)$ & 0.0022 \\
\hline Ruminococcaceae (560535) OTU log relative abundance $\ddagger$ & NA &.. & NA &.$\cdot$ & $1.43(1.02-2 \cdot 00)$ & 0.036 \\
\hline Sutterella (589923) OTU log relative abundanceł & NA &.. & NA &.. & $0.81(0.65-1.00)$ & 0.049 \\
\hline \multicolumn{7}{|l|}{ Model characteristics } \\
\hline AUC & $0.70(0.65-0.75)$ &.. & $0.68(0.61-0.75)$ &. & $0.75(0.68-0.83)$ &.. \\
\hline Cross-validation AUC & $0.63(0.57-0.69)$ &.$\cdot$ & $0.49(0.40-0.58)$ &.$\cdot$ & $0.72(0.64-0.80)$ &.$\cdot$ \\
\hline $\mathrm{R}^{2}$ & $0 \cdot 16$ &.. & 0.17 & .. & 0.29 &. \\
\hline Sensitivity & $47 \%(39-55)$ &.. & $70 \%(57-80)$ & .. & $49 \%(38-61)$ & $\cdot$. \\
\hline Specificity & $77 \%(71-82)$ &.$\cdot$ & $60 \%(50-69)$ & .. & $78 \%(70-85)$ &.. \\
\hline Positive predictive value & $56 \%(47-64)$ & .. & $53 \%(42-63)$ & .. & $58 \%(46-71)$ &.. \\
\hline Negative predictive value & $70 \%(65-76)$ &.$\cdot$ & $76 \%(65-84)$ &.. & $71 \%(62-79)$ &.. \\
\hline \multicolumn{7}{|l|}{ Clinical plus biological model vs clinical model $\$$} \\
\hline Likelihood ratio test & NA & NA & NA & NA &.. & 0.00037 \\
\hline Comparison of AUC & NA & NA & NA & NA &.. & 0.014 \\
\hline $\begin{array}{l}\text { Hosmer-Lemeshow goodness-of-fit test, median multiple } \\
\text { imputation } p \text { value (proportion of imputations with } p>0.05 \text { ) }\end{array}$ &.. & $0.42(100 \%)$ &.. & $0.99(100 \%)$ &.. & $0.25(100 \%)$ \\
\hline
\end{tabular}

Baseline predictors are odds ratio, unless otherwise stated, with 95\% Cls in parentheses when appropriate. Missing covariate data imputed via multiple imputation. Clinical parameters selected by LASSO. Sensitivity, specificity, positive predictive value, and negative predictive value were based on a predicted probability of 0.50 . The predicted probability of corticosteroid-free remission for a patient with a PUCAI score of $<45$, haemoglobin $\geq 10 \mathrm{~g} / \mathrm{dL}$, and week 4 remission is 0.60 (for equation see appendix). Model fitting identified an interaction between haemoglobin concentration and week 4 remission ( $\mathrm{p}=0.042$ ), which led to the final model with a predictor of haemoglobin $\geq 10 \mathrm{~g} / \mathrm{dL}$ in the absence of week 4 remission. PUCAl=Pediatric Ulcerative Colitis Activity Index. NA=not applicable. OTU=operational taxonomic unit. AUC=area under the curve. LASSO=least absolute shrinkage and selection operator. ${ }^{*}$ The per-protocol population excludes participants who discontinued the study without additional therapy or colectomy or who had protocol violations. †The clinical model was run on the subset of participants with biological data. Clinical model factors with $p>0.05$ in this smaller cohort were removed from the model. $\neq$ Log relative abundance was calculated by taking the log base 10 of the OTU plus half of the minimum value. \$Comparison of the clinical plus biological model with clinical model in the subset of patients with biological data.

Table 3: Multivariable logistic regression models of week 52 corticosteroid-free remission in the per-protocol population

concentrations of less than $10 \mathrm{~g} / \mathrm{dL}$, low serum 25(OH)D concentration, and low eosinophil count in rectal biopsy samples were also predictive of escalation to antiTNF $\alpha$ therapy in patients with moderate-to-severe disease (table 4). A model including these factors showed good discriminatory power, with an AUC of $0.78(95 \% \mathrm{CI}$ $0 \cdot 72-0 \cdot 84)$, a cross-validated AUC of $0.75(0 \cdot 68-0 \cdot 82)$, sensitivity of $58 \%$ (95\% CI $47-68)$, and specificity of $85 \%$ (95\% CI 78-92; table 4; appendix). A post-hoc model with week 12 corticosteroid-free remission instead of week 4 remission had a similar AUC of 0.78 (95\% CI $0 \cdot 72-0 \cdot 85)$, sensitivity of $59 \%$ (95\% CI 48-69), and specificity of $85 \%$ ( $95 \%$ C I 78-92). B ecause total Mayo score, serum $25(\mathrm{OH}) \mathrm{D}$ concentration, and rectal biopsy sample eosinophil count were not collected in the IBD Registry study, ${ }^{7}$ we were not able to use these data to validate this secondary model. The association between the probability of escalation to anti-TNF $\alpha$ therapy based on the clinical model and the transport and antimicrobial gene signature is shown in the appendix. Patients with a lower transport and antimicrobial gene signature PC1 were more likely to escalate to anti-TNF $\alpha$ therapy (appendix). The addition of biological data on transport and antimicrobial gene signature and the abundance of an Oscillospira species to the model improved the model fit with an AUC of 0.88 (95\% CI 0.81-0.94; $\mathrm{p}=0.016)$, a cross-validated AUC of $0 \cdot 84(0 \cdot 76-0 \cdot 91)$, sensitivity of $66 \%$ (95\% CI $52-80)$, and specificity of $83 \%$ (95\% CI 74-91) in the subset with transcriptomic and microbial data $(\mathrm{n}=118$; figure $3 \mathrm{C}$, table 4). Patients with low transport gene expression and Oscillospira concentrations and high antimicrobial gene expression had an increased likelihood of escalating to anti-TNF $\alpha$ therapy (table 4).

\section{Discussion}

In this study, despite utilisation of standardised initial therapy with mesalazine or corticosteroid, a minority of children with ulcerative colitis achieved the ideal clinical outcome of corticosteroid-free remission with mesalazine alone at 1 year after diagnosis. 19\% of participants required additional medical therapy with immunomodulators such as thiopurines, $31 \%$ were given antiTNF $\alpha$ therapy, and $6 \%$ required a colectomy within 1 year of diagnosis. These clinical outcomes are consistent with a previously reported ${ }^{1}$ incidence of $8 \%$ colectomy among children newly diagnosed with ulcerative colitis. 


\begin{tabular}{|c|c|c|c|c|c|c|}
\hline & \multicolumn{2}{|c|}{$\begin{array}{l}\text { All patients in clinical model } \\
(n=232 ; 94[41 \%] \text { events) }\end{array}$} & \multicolumn{4}{|c|}{ Patients with biological data $†$ ( $n=118 ; 43[39 \%]$ events) } \\
\hline & \multirow[t]{2}{*}{ Estimate } & \multirow[t]{2}{*}{ pvalue } & \multicolumn{2}{|l|}{ Clinical model } & \multicolumn{2}{|c|}{ Clinical plus biological model } \\
\hline & & & Estimate & p value & Estimate & p value \\
\hline \multicolumn{7}{|l|}{ Baseline predictors } \\
\hline Total Mayo score $\geq 11$ & $4.38(2 \cdot 25-8 \cdot 54)$ & 0.0002 & NA & .. & NA & .. \\
\hline Rectal biopsy eosinophil peak count $>32$ per hpf & $0.48(0.25-0.92)$ & 0.027 & NA &.$\cdot$ & NA &.$\cdot$ \\
\hline Higher 25-hydroxyvitamin D concentration, per increase in category $\ddagger$ & $0.60(0.37-0.96)$ & 0.034 & $0.29(0.11-0.74)$ & 0.010 & $0.31(0.14-0.71)$ & 0.0052 \\
\hline Haemoglobin $\geq 10 \mathrm{~g} / \mathrm{dL}$ & $0.50(0.26-0.95)$ & 0.033 & $0.39(0 \cdot 20-0 \cdot 76)$ & 0.0063 & $0.33(0.11-0.94)$ & 0.038 \\
\hline Week 4 remission & $0.36(0.19-0.68)$ & 0.0015 & $0.16(0.06-0.41)$ & 0.00012 & $0.21(0.07-0.58)$ & 0.0029 \\
\hline Transport and antimicrobial gene signature & NA & .. & NA & .. & $0.31(0.16-0.61)$ & 0.00063 \\
\hline Oscillospira (581079) OTU log relative abundance $\$$ & NA & .. & NA & .. & $0.64(0.44-0.92)$ & 0.018 \\
\hline \multicolumn{7}{|l|}{ Model characteristics } \\
\hline AUC & $0.78(0.72-0.84)$ & .. & $0.80(0.72-0.88)$ & .. & $0.88(0.81-0.94)$ & .. \\
\hline Cross-validated AUC & $0.75(0.68-0.82)$ & .. & $0.75(0.65-0.84)$ & .. & $0.84(0.76-0.91)$ & .. \\
\hline$R^{2}$ & 0.32 & .. & $0 \cdot 37$ &. & 0.52 &.. \\
\hline Sensitivity & $58 \%(47-68)$ & .. & $63 \%(49-77)$ &. & $66 \%(52-80)$ &.$\cdot$ \\
\hline Specificity & $85 \%(78-92)$ & .. & $82 \%(73-91)$ & .. & $83 \%(74-91)$ & .. \\
\hline Positive predictive value & $73 \%(61-84)$ & .. & $69 \%(55-83)$ & .. & $71 \%(57-85)$ & .. \\
\hline Negative predictive value. & $75 \%(68-82)$ & .. & $78 \%(68-87)$ &. & $79 \%(70-89)$ &. \\
\hline \multicolumn{7}{|l|}{ Clinical plus biological model vs clinical model $\Phi$} \\
\hline Likelihood ratio test & NA & NA & NA & .. & .. & 0.00004 \\
\hline Comparison of AUC & NA & NA & NA &. &. & 0.016 \\
\hline $\begin{array}{l}\text { Hosmer-Lemeshow goodness-of-fit test, median multiple imputation } \\
\text { p value (proportion of imputations with } p>0.05 \text { ) }\end{array}$ & .. & $0.43(99 \%)$ & .. & $0.96(100 \%)$ & .. & $0.53(100 \%)$ \\
\hline \multicolumn{7}{|c|}{ 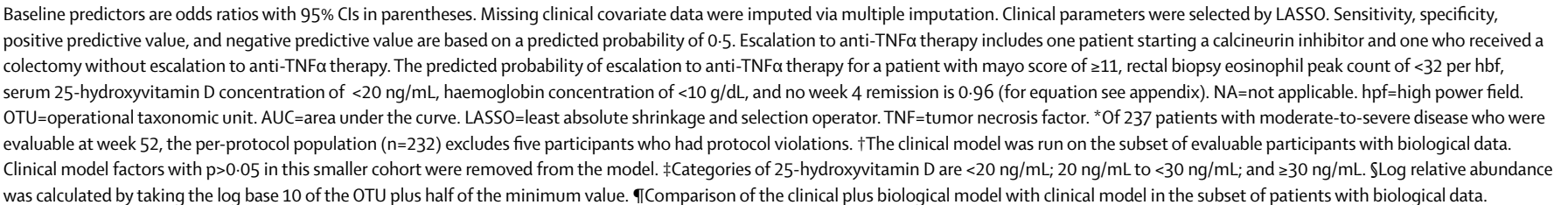 } \\
\hline
\end{tabular}

Table 4: Multivariable logistic regression models of escalation to anti-TNF $\alpha$ therapy by week 52 for patients with moderate-to-severe disease

Although in our study severe clinical presentations were associated with worse outcomes, some patients with severe disease went on to achieve week 52 corticosteroidfree remission and some with initially mild disease required escalation of therapy.

An important finding of our study was the importance of early response to mesalazine or corticosteroids and achieving clinical remission by week 4 with these therapies as predictors of outcome at week 52, and we replicated this observation in a large independent cohort. A previous retrospective report ${ }^{22}$ of children with ulcerative colitis who underwent a similar treatment regimen suggested that PUCAI score at 3 months was an important predictor of corticosteroid-free remission at 1 year. Early prediction of disease course would be preferable and could lead to more timely introduction of additional medical therapy beyond mesalazine or corticosteroids, especially in those who presented with moderate-to-severe disease. Recent guidelines from the European Crohn's and Colitis Organization (ECCO) and European Society for Paediatric Gastroenterology
Hepatology and Nutrition (ESPGHAN) recommend that children who present with acute severe colitis who respond to intravenous corticosteroids can generally be transitioned to thiopurines. ${ }^{23}$ However, our data suggest that such a treatment approach might not be necessary in all patients if clinical remission is achieved by 4 weeks, and that these children should be given an opportunity to show their ability to achieve early clinical remission and transition to mesalazine before being routinely placed on thiopurines or anti-TNF $\alpha$ drugs.

We also sought to identify biological predictors of disease course. Patients with $25(\mathrm{OH}) \mathrm{D}$ concentrations of less than $20 \mathrm{ng} / \mathrm{mL}$ before treatment were more likely to escalate to anti-TNF $\alpha$ therapy than those with concentrations of $20 \mathrm{ng} / \mathrm{mL}$ or higher before treatment. These findings are consistent with a murine study ${ }^{24}$ that showed the beneficial effects of vitamin D on the function of the epithelial barrier of the gut and inflammatory immune responses and previous data in adults with ulcerative colitis showing worse clinical outcomes were associated with low 25(OH)D concentrations. ${ }^{6}$ Patients 
with rectal eosinophil counts of less than 32 per highpower field before treatment were more likely to escalate to anti-TNF $\alpha$ therapy than those with higher counts, which is consistent with data from adult patients with ulcerative colitis that showed worse outcomes were associated with decreased rectal eosinophils counts. ${ }^{25}$ These data suggest that incorporation of baseline $25(\mathrm{OH}) \mathrm{D}$ concentrations and rectal eosinophil counts into assessment of the likelihood of escalation to anti-TNF $\alpha$ therapy in ulcerative colitis could be helpful, and might prioritise clinical trials targeting these pathways.

Shifts in the gut microbial community and host intestinal gene expression have been considered in predicting the likelihood of response to biological therapies. ${ }^{26-29}$ Little data regarding response to initial therapy with mesalazine and corticosteroid exist. We found that decreased expression of genes that encode ion channels and transporters and increased expression of antimicrobial peptides were associated with an increased likelihood of escalation to anti-TNF $\alpha$ therapy. The epithelial transport gene signature was enriched for guanylin activity, which ameliorates murine colitis..$^{30}$ Although decreased expression of $\alpha$-defensin by Paneth cells has been implicated in the pathogenesis of ileal Crohn's Disease, ${ }^{31}$ for the first time to our knowledge, we linked up-regulated rectal expression of $\alpha$-defensin to an increased refractory course in patients with ulcerative colitis who were treatment naive. In this context, $\alpha$-defensins might contribute to colonic injury via direct cytotoxic activity and recruitment of inflammatory leukocytes. ${ }^{28}$ We found 28 (with a false discovery rate of $<0.05)$ microbial operational taxonomic units were linked to clinical outcomes, with the majority from the Clostridiales order. Decreased expression of Clostridiales at diagnosis was associated with escalation to anti-TNF $\alpha$ therapy. This observation is consistent with studies linking these taxa to improved function of the colonic barrier and anti-inflammatory regulatory $\mathrm{T}$ cells. ${ }^{21}$

Our study has several limitations and notable strengths. Patient enrolment was not population based and so we cannot exclude some degree of selection bias. However, the 29 study centres were representative of paediatric ulcerative colitis care in North America. Only 69 (16\%) part icipants self-identified a n non-white, and s o our results might not be generalisable to a non-white patient population. The complexity and expense of completing this study did not allow us to develop a similar validation cohort with standardised therapy and biological measures. Gene expression and microbiome data were available for only a subset of participants who strictly followed the treatment regimen; therefore, a validation study will be required. Furthermore, our results should be interpreted with caution because statistical tests were done at a two-sided $\alpha$ level of 0.05 with no adjustment. A strength of our study that supports the generalisability of our findings is that our initial standardised treatment regimen is in harmony with recent ECCO and North American Society for Pediatric Gastroenterology, Hepatology and Nutrition (NASPGHAN) guidelines for the treatment of paediatric ulcerative colitis $^{23,32}$ and we prospectively enrolled a large number of children with varying disease severity. Our primary outcome incidence of corticosteroid-free remission on mesalazine only is similar to that reported previously from an uncontrolled observational registry of children who were newly diagnosed with ulcerative colitis, ${ }^{7}$ and we replicated our main clinical model within this group.

Physicians, patients, and families usually seek early information that facilitates informed choices when balancing the risks and benefits of additional medical therapy. Our data will have an immediate clinical effect on the treatment of children with ulcerative colitis. By week 4 after initial therapy with mesalazine or corticosteroid, many children declared their treatment response (ie, achieved clinical remission). Our validated clinical model including baseline severity coupled with week 4 remission has good specificity, which will minimise the number of false positive results in providing improved confidence to guide treatment decisions. Readily available clinical indices of severity, rectal eosinophil counts, and 25(OH)D concentrations can be used when considering use of antiTNF $\alpha$ therapy. Additional studies on rectal gene expression and microbial factors in the gut could improve prediction of response to initial therapy, identify novel therapeutic targets, and help develop personalised approaches to improve outcomes in children with ulcerative colitis.

\section{Contributors}

The PROTECT steering committee (JSH, SDT, MAM, SK, TW, and LAD) conceived and designed the study, analysed the data, and wrote the first draft of the manuscript. NG, YH, RK, MS, AM, UMM, GG, RJX, SV, BS and JW generated and analysed the data and participated in drafting the manuscript. FAS, MD, VT, MHC, KH, and KLS gathered and analysed data and participated in drafting the manuscript. DRM, BB, AMG, NSL, CGS, DJK, JM, SSB, JR, RNB, AP, MP, AO, MH, JN, MO-H, PAR, JSt, $\mathrm{DZ}, \mathrm{SLG}, \mathrm{BS}, \mathrm{KB}, \mathrm{PW}, \mathrm{DM}$, JE, and MDK recruited patients, gathered data and participated in manuscript revision. JSe provided study oversight and participated in manuscript revision. All authors had access to study data and approved the decision to submit the manuscript.

\section{Declaration of interests}

JSH has served on an advisory board for Janssen and is acting as a consultant for AbbVie, Takeda, Lilly, Boehringer-Ingelheim, Allergan, Pfizer, Receptos, and AstraZeneca. SDT has been a member of an independent data monitoring committee for Lycera Corporation. AMG has received research support from AbbVie; been a consultant for AbbVie, Celgene, Janssen, Lilly, Pfizer, and Takeda; and been a speaker for AbbVie, Janssen, and Shire. NSL has been a consultant for AbbVie. CGS has been a consultant for AbbVie. DJK has received research support from Genentech and Takeda. JM has been a consultant for Janssen, Celgene, and Lilly. JR has been a consultant for AbbVie, Celgene, Janssen, Luitpold, and Pfizer, and received grant funding from Janssen and AbbVie. AP has participated in speakers bureaus AbbVie and Janssen. AO has been a member of an advisory board for Janssen and AbbVie, and received research support from Lilly, AbbVie, Janssen, Takeda, and Celgene. MH has received research grants from Genentech, AbbVie, Shire, Takeda, Mallinkrodt, Janssen, and Gilead. MO-H has received research grants from Abbott Immunology and Janssen and been a consultant for Hoffman LaRoche. PAR has been a consultant for Shire and Leutpold; been a speaker for AbbVie; and received research support from TechLab. JSt has been a consultant and speaker for AbbVie. DZ has participated on speakers bureaus for AbbVie and been a consultant for 11 Health and Technologies and Vitality Biopharma. MDK has been a consultant for AbbVie, Janssen, GlaxoSmithKline, and Pfizer. MHC has 
been a consultant for Shire, Regeneron, Receptos, and Allakos, and has research contracts with Shire and Regeneron. MD has been a consultant for Prometheus Laboratories, AbbVie, Janssen, Takeda, Pfizer, Celgene, UCB, Boehringer Ingelheim, Lilly, Gilead, and Allergan. SK has been a consultant for Janssen and UCB. LAD has received grant support from AbbVie and Janssen. All other authors declare no competing interests.

\section{Data sharing}

Data from the PROTECT study will be available from the US National Institutes of Diabetes, and Digestive and Kidney Diseases data repository (https://repository.niddk.nih.gov/home/). Requestors can apply for access via this website and must have approval from their insitution's review board.

\section{Acknowledgments}

We thank Frank Hamilton and Stephen James from the US National Institute for Diabetes and Digestive and Kidney Diseases (NIDDK) for their guidance; Curtis Huttenhower, Tiffany Poon, and Hera Vlamakis from The Broad Institute for their assistance; and William Faubion for his role as safety monitor. We also thank the Crohn's and Colitis Foundation (New York, NY, USA) for their invaluable support for this study. The study investigators thank Shire Pharmaceuticals for providing Pentasa (mesalazine) for this study (IND 111863), the research coordinators at the study sites for their tireless attention, and the patients and families who agreed to participate in this important study. Support for this study was provided by NIDDK (5U01DK095745 and P30 DK078392), Integrative Morphology and Gene Expression Cores, NIDDK (DK043351 and AT009708, CSIBD DK043351), and Center for Microbiome Informatics and Therapeutics at Massachusetts Institute of Technology.

\section{References}

1 Gower-Rousseau C, Dauchet L, Vernier-Massouille G, et al. The natural history of pediatric ulcerative colitis: a population-based cohort study. Am J Gastroenterol 2009; 104: 2080-88.

2 Turner D, Mack D, Leleiko N, et al. Severe pediatric ulcerative colitis: a prospective multicenter study of outcomes and predictors of response. Gastroenterology 2010; 138: 2282-91.

3 Hyams JS, Davis P, Grancher K, Lerer T, Justinich CJ, Markowitz J. Clinical outcome of ulcerative colitis in children. J Pediatr 1996; 129: 81-88.

4 Melson JE, Giusto D, Kwasny M, Eichenseer P, Jakate S, Keshavarzian A. Histopathology predictors of medically refractory ulcerative colitis. Dis Colon Rectum 2010; 53: 1280-86.

5 Haritunians T, Taylor KD, Targan SR, et al. Genetic predictors of medically refractory ulcerative colitis. Inflamm Bowel Dis 2010; 16: $1830-40$.

6 Gubatan J, Mitsuhashi S, Zenlea T, Rosenberg L, Robson S, Moss AC. Low serum vitamin D during remission increases risk of clinical relapse in patients with ulcerative colitis. Clin Gastroenterol Hepatol 2017; 15: 240-46.

7 Zeisler B, Lerer T, Markowitz J, et al. Outcome following aminosalicylate therapy in children newly diagnosed as having ulcerative colitis. J Pediatr Gastroenterol Nutr 2013; 56: 12-18.

8 Bousvaros A, Antonioli DA, Colletti RB, et al. Differentiating ulcerative colitis from Crohn disease in children and young adults: report of a working group of the North American Society for Pediatric Gastroenterology, Hepatology, and Nutrition and the Crohn's and Colitis Foundation of America. J Pediatr Gastroenterol Nutr 2007; 44: 653-74.

9 Hyams JS, Davis S, Mack DR, et al. Factors associated with early outcomes following standardised therapy in children with ulcerative colitis (PROTECT): a multicentre inception cohort study. Lancet Gastroenterol Hepatol 2017; 2: 855-68.

10 Turner D, Otley AR, Mack D, et al. Development, validation, and evaluation of a pediatric ulcerative colitis activity index: a prospective multicenter study. Gastroenterology 2007; 133: 423-32.

11 Schroeder KW, Tremaine WJ, Ilstrup DM. Coated oral 5 -aminosalicylic acid therapy for mildly to moderately active ulcerative colitis. A randomized study. N Engl J Med 1987; 317: 1625-29.

12 Boyle B, Collins MH, Wang Z, et al. Histologic correlates of clinical and endoscopic severity in children newly diagnosed with ulcerative colitis. Am J Surg Pathol 2017; 41: 1491-98.
13 Burri E, Manz M, Rothen C, Rossi L, Beglinger C, Lehmann FS Monoclonal antibody testing for fecal calprotectin is superior to polyclonal testing of fecal calprotectin and lactoferrin to identify organic intestinal disease in patients with abdominal discomfort. Clin Chim Acta 2013; 416: 41-47.

14 Venkateswaran S, Prince J, Cutler DJ, et al. Enhanced contribution of HLA in pediatric onset ulcerative colitis. Inflamm Bowel Dis 2018 24: 829-38.

15 Haberman Y, Tickle TL, Dexheimer PJ, et al. Pediatric Crohn disease patients exhibit specific ileal transcriptome and microbiome signature. J Clin Invest 2014; 124: 3617-33.

16 Tuerk A, Wiktorin G, Güler S. Mixture models reveal multiple positional bias types in RNA-Seq data and lead to accurate transcript concentration estimates. PLoS Comput Biol 2017; 13: e1005515.

17 Gevers D, Kugathasan S, Denson LA, et al. The treatment-naive microbiome in new-onset Crohn's disease. Cell Host Microbe 2014; 15: 382-92.

18 Friedman J, Hastie T, Tibshirani R. Regularization paths for generalized linear models via coordinate descent. J Stat Softw 2010; 33: $1-22$.

19 Haberman Y, Karns R, Dexheimer PJ, et al. Ulcerative colitis mucosal transcriptomes reveal mitochondriopathy and personalized mechanisms underlying disease severity and treatment response. Nat Commun 2019; 10: 38

20 Kugathasan S, Denson LA, Walters TD, et al. Prediction of complicated disease course for children newly diagnosed with Crohn's disease: a multicentre inception cohort study. Lancet 2017; 389: 1710-18.

21 Atarashi K, Tanoue T, Oshima K, et al. Treg induction by a rationally selected mixture of Clostridia strains from the human microbiota. Nature 2013; 500: 232-36.

22 Schechter A, Griffiths C, Gana JC, et al. Early endoscopic, laboratory and clinical predictors of poor disease course in paediatric ulcerative colitis. Gut 2015; 64: 580-88.

23 Turner D, Ruemmele FM, Orlanski-Meyer E, et al. Management of paediatric ulcerative colitis, part 2: acute severe colitisan evidence-based consensus guideline from the European Crohn's and Colitis Organization and the European Society of Paediatric Gastroenterology, Hepatology and Nutrition. J Pediatr Gastroenterol Nutr 2018; 67: 292-310.

24 Liu W, Chen Y, Golan MA, et al. Intestinal epithelial vitamin D receptor signaling inhibits experimental colitis. J Clin Invest 2013 123: 3983-96.

25 Heatley RV, James PD. Eosinophils in the rectal mucosa. A simple method of predicting the outcome of ulcerative proctocolitis? Gut 1979; 20: 787-91.

26 Ananthakrishnan AN, Luo C, Yajnik V, et al. Gut microbiome function predicts response to anti-integrin biologic therapy in inflammatory bowel diseases. Cell Host Microbe 2017; 21: 603-10.

27 Arijs I, Li K, Toedter G, et al. Mucosal gene signatures to predict response to infliximab in patients with ulcerative colitis. Gut 2009; 58: 1612-19.

28 Arijs I, De Hertogh G, Lemaire K, et al. Mucosal gene expression of antimicrobial peptides in inflammatory bowel disease before and after first infliximab treatment. PLoS One 2009; 4: e7984.

29 West NR, Hegazy AN, Owens BMJ, et al. Oncostatin M drives intestinal inflammation and predicts response to tumor necrosis factor-neutralizing therapy in patients with inflammatory bowel disease. Nat Med 2017; 23: 579-89.

30 Harmel-Laws E, Mann EA, Cohen MB, Steinbrecher KA. Guanylate cyclase $\mathrm{C}$ deficiency causes severe inflammation in a murine model of spontaneous colitis. PLoS One 2013; 8: e79180.

31 VanDussen KL, Liu TC, Li D, et al. Genetic variants synthesize to produce paneth cell phenotypes that define subtypes of Crohn's disease. Gastroenterology 2014; 146: 200-09.

32 Turner D, Ruemmele FM, Orlanski-Meyer E, et al. Managemen of paediatric ulcerative colitis, part 1: ambulatory carean evidence-based guideline from European Crohn's and Colitis Organization and European Society of Paediatric Gastroenterology, Hepatology and Nutrition. J Pediatr Gastroenterol Nutr 2018; 67: 257-91. 